\title{
A Method of Speed Estimation with Stator Resistance Online Identification Based on MRAS
}

\author{
Tong Jun ${ }^{1, a}$, Sun Lei ${ }^{1, b}$, Cao Hao-xiang ${ }^{1, c}$ \\ ${ }^{1}$ School of Electrical and Control Engineering, Xi'an University \\ of Science and Technology, Xi'an 710054, China. \\ a642880570@qq.com, b2422457037@qq.com, '1004716367@qq.com
}

\begin{abstract}
Keywords: induction motor, speed sensor-less, speed estimation, stator resistance online identification, model reference adaptive system(MRAS).
\end{abstract}

\begin{abstract}
It is important to accurately estimate the speed for induction motor speed sensor-less control technology. According to the drawbacks of traditional model reference adaptive system (MRAS), this paper presents an improved estimation model, which can effectively eliminate the DC drift and the initial value problems caused by pure Integrator. On this basis, it analyzes the influence of variable stator resistance on the speed estimation and makes stator resistance online identification to update stator resistance changed by temperature and ensure the accuracy of speed estimation. The simulation and experimental results show the rationality and effectiveness of the method.
\end{abstract}

\section{Introduction}

In order to improve the speed performance of the induction motor, but it not only increases the cost, but also requires strict installation environment which is uneasy to maintain. Therefore, the research of speed sensor-less control technology has become a hot topic at home and abroad.

There are many kinds of the motor speed estimation methods, model reference adaptive system (MRAS) is widely used due to its easier algorithm and higher estimation precision. Traditional MRAS builds speed estimation algorithm by rotor magnetic flux linkage. In $\alpha-\beta$ coordinate system, the voltage model of rotor magnetic flux linkage is regarded as reference model and the current model is regarded as adjustable mode. The principle is clear and practical, as in [1]. But the pure integral part of the voltage model has the problems of initial value setting and DC integration drift, which affect the accuracy of observation and the stability of the system, as in [2].

Based on traditional MRAS speed estimation model, low-pass filter is used to replace the original pure integral element, which can effectively eliminate integral initial value and DC drift to improve the system's ability to resist disturbance. Moreover, a speed estimation method is proposed to estimate the stator resistance, which can avoid speed estimation error caused by temperature variation. Finally, simulations and experiments are carried out to verify the above conclusions.

\section{Traditional MRAS Speed Estimation Method}

The voltage model and current model of rotor magnetic flux linkage in $\alpha-\beta$ coordinate system are shown as formula (1) and (2):

$$
\left\{\begin{array}{l}
\psi_{r \alpha}=\frac{L_{r}}{L_{m}}\left[\int\left(u_{s \alpha}-R_{s} i_{s \alpha}\right) d t-\sigma L_{s} i_{s \alpha}\right] \\
\psi_{r \beta}=\frac{L_{r}}{L_{m}}\left[\int\left(u_{s \beta}-R_{s} i_{s \beta}\right) d t-\sigma L_{s} i_{s \beta}\right]
\end{array}\right.
$$

$$
\left\{\begin{array}{l}
p \hat{\psi}_{r \alpha}=-\frac{1}{T_{r}} \hat{\psi}_{r \alpha}-\omega_{r} \hat{\psi}_{r \beta}+\frac{L_{m}}{T_{r}} i_{s \alpha} \\
p \hat{\psi}_{r \beta}=-\frac{1}{T_{r}} \hat{\psi}_{r \beta}+\omega_{r} \hat{\psi}_{r \alpha}+\frac{L_{m}}{T_{r}} i_{s \beta}
\end{array}\right.
$$

where $\psi_{r \alpha}$ and $\psi_{r \beta}$ represent rotor magnetic flux linkage $\alpha$-axis and $\beta$-axis components in the voltage model; $\hat{\psi}_{r \alpha}$ and $\hat{\psi}_{r \beta}$ represent rotor magnetic flux linkage $\alpha$-axis and $\beta$-axis components in the current model; $u_{s \alpha}$ and $u_{s \beta}$ represent stator voltage $\alpha$-axis and $\beta$-axis components; $i_{s \alpha}$ and $i_{s \beta}$ represent stator current $\alpha$-axis and $\beta$-axis components; $R_{s}$ and $R_{r}$ represent stator and rotor resistance; $L_{s}, L_{r}$ and $L_{m}$ represent stator inductance, rotor inductance and mutual 
inductance; $\sigma$ represents magnetic flux leakage coefficient, which is given by $\sigma=1-L_{m}^{2} /\left(L_{s} L_{r}\right) ; T_{r}$ represents rotor time constant, which is given by $T_{r}=L_{r} / R_{r} ; \omega_{r}$ represents rotor speed.

From formulas (1) and (2), we can see that two models have the same output, rotor magnetic flux linkage. Voltage model has nothing to do with speed, but current model is related to rotor speed. Voltage model is regarded as reference model and current model is regarded as adjustable model. When the rotor magnetic flux linkage error of the two models becomes zero, it is considered that the estimated speed has already been equal to the actual speed. Because the voltage model is a pure integrator, integral operation not only exists the initial value setting problem, but also inevitably contains DC component in the actual measurements of voltage and current signals. It will affect stability of the system and accuracy of calculation. Thus, the formula (1) can not be used for the actual calculation(e.g.[3][4]).

\section{Improved MRAS Speed Estimation Method}

In order to solve the drawbacks of traditional MRAS in speed estimation, the low-pass filter is used to instead of pure integral. The improved voltage model can be equivalent to the output of the original model connected with a high-pass filter. Given that high-pass filter will cause a phase lag, a same high-pass filter is added to the current model. Improved voltage model and current model are shown as formula (3) and (4) :

$$
\left\{\begin{array} { l } 
{ \psi _ { r \alpha } = \frac { s L _ { r } } { ( s + 1 / T ) L _ { m } } [ \int ( u _ { s \alpha } - R _ { s } i _ { s \alpha } ) d t - \sigma L _ { s } i _ { s \alpha } ] } \\
{ \psi _ { r \beta } = \frac { s L _ { r } } { ( s + 1 / T ) L _ { m } } [ \int ( u _ { s \beta } - R _ { s } i _ { s \beta } ) d t - \sigma L _ { s } i _ { s \beta } ] }
\end{array} \left(\begin{array}{l}
p \hat{\psi}_{r \alpha}=\frac{s}{s+1 / T}\left(-\frac{1}{T_{r}} \hat{\psi}_{r \alpha}-\omega_{r} \hat{\psi}_{r \beta}+\frac{L_{m}}{T_{r}} i_{s \alpha}\right) \\
p \hat{\psi}_{r \beta}=\frac{s}{s+1 / T}\left(-\frac{1}{T_{r}} \hat{\psi}_{r \beta}+\omega_{r} \hat{\psi}_{r \alpha}+\frac{L_{m}}{T_{r}} i_{s \beta}\right)
\end{array}\right.\right.
$$

The error formula of rotor flux linkage is defined as:

$$
\left[\begin{array}{c}
e_{r \alpha} \\
e_{r \beta}
\end{array}\right]=\left[\begin{array}{l}
\hat{\psi}_{r \alpha} \\
\hat{\psi}_{r \beta}
\end{array}\right]-\left[\begin{array}{c}
\psi_{r \alpha} \\
\psi_{r \beta}
\end{array}\right]
$$

Applying Popov super stability theory constructs the adaptive law of speed:

$$
\hat{\omega}_{r}=k_{p 1} \int_{0}^{t}\left(e_{r \beta} \hat{\psi}_{r \alpha}-e_{r \alpha} \hat{\psi}_{r \beta}\right) d \tau+k_{p}\left(e_{r \beta} \hat{\psi}_{r \alpha}-e_{r \alpha} \hat{\psi}_{r \beta}\right)=k_{i 1} \int_{0}^{t}\left(\psi_{r \beta} \hat{\psi}_{r \alpha}-\psi_{r \alpha} \hat{\psi}_{r \beta}\right) d \tau+k_{p}\left(\psi_{r \beta} \hat{\psi}_{r \alpha}-\psi_{r \alpha} \hat{\psi}_{r \beta}\right)
$$

Where $k_{p 1}$ represents the proportion factor, $k_{i 1}$ represents the integral factor. Converse to $\mathrm{S}$ domain, which is expressed as:

$$
\hat{\omega}_{r}=\left(k_{p 1}+\frac{k_{s 1}}{s}\right)\left(\psi_{r \beta} \hat{\psi}_{r \alpha}-\psi_{r \alpha} \hat{\psi}_{r \beta}\right)
$$

Thus, a complete model reference adaptive system can be formed to achieve the speed estimation of three-phase induction motor by the formula (3), (4) and (7). The improved MRAS speed estimation schematic diagram is shown in Fig.1. The speed estimation can be obtained by choosing the appropriate PI parameters. According to the Popov super stability theory, the adaptive system is asymptotically convergent and stable.

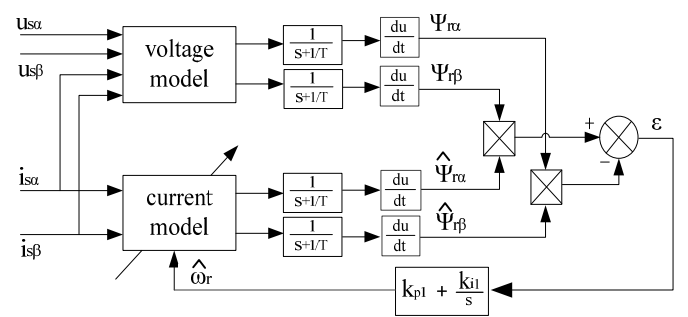

Fig. 1 Schematic diagram of improved MRAS speed estimation 


\section{Stator Resistance Identified Online MRAS Speed Estimation Method}

\subsection{The Influence of Stator Resistance on Speed Estimation}

In the motor running process, the motor stator resistance is not constant, which will rise with the increase of motor temperature, as in [5]. When the stator resistance changes, the rotor magnetic flux linkage in voltage model will turn into:

$$
\psi_{r \alpha}^{\prime}=\frac{L_{r}}{L_{m}}\left[\int\left(u_{s \alpha}-R_{s}^{\prime} i_{s \alpha}\right) d t-\sigma L_{s} i_{s \alpha}\right]=\psi_{r \alpha}-\frac{L_{r}}{L_{m}} \int \Delta R_{s} i_{s \alpha} d t
$$

Where $\psi_{r \alpha}^{\prime}$ represents the actual rotor magnetic flux linkage; $R_{s}^{\prime}$ represents the actual stator resistance; $\Delta R_{s}$ represents the stator resistance error.

From the formula (8), it can be seen that the stator resistance error will cause inaccurate of the rotor magnetic flux linkage observation in voltage model and couple with integral form so that the stator resistance error is accumulated continuously. It will decrease accuracy of speed estimation and even diverge.

\subsection{MRAS Speed Estimation of Stator Resistance Online Identification}

Given that the stator resistance variation can not be ignored in the speed estimation, this paper presents a induction motor speed estimation scheme based on stator resistance online identification. The principle block diagram is shown in Fig.2.

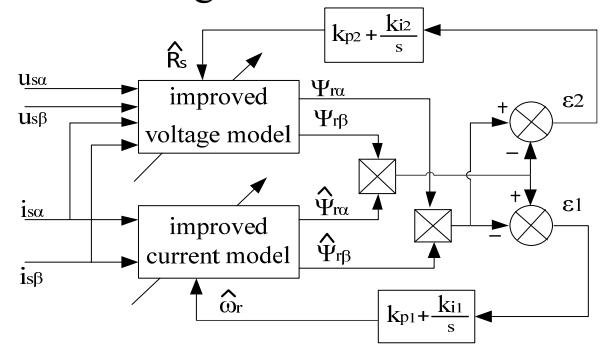

Fig. 2 MRAS speed estimation of stator resistance online identification block diagram

Stator resistance has nothing to do with the improved current model, which is related to voltage model, so improved current model can be used as the reference model, improved the voltage model as the adjustable model, as in [6]. The adaptive law of stator resistance is constructed based on the Popov's super stability theory:

$$
\hat{R}_{s}=k_{p 2}\left(\psi_{r \alpha} \hat{\psi}_{r \beta}-\psi_{r \beta} \hat{\psi}_{r \alpha}\right)+k_{i 2} \int_{0}^{T}\left(\psi_{r \alpha} \hat{\psi}_{r \beta}-\psi_{r \beta} \hat{\psi}_{r \alpha}\right) d t
$$

Where $k_{p 2}$ represents the proportion factor, $k_{i 2}$ represents the integral factor.

The key of this design is how to coordinate the stator resistance identification and rotor speed identification simultaneously. For infrequent starting motor, the system work can be divided into two stages. The first stage is the speed estimation. When the motor is initially started, the body temperature is basically unchanged, stator resistance in the voltage model can be set to its initial value, the speed is stable after a few seconds. The second stage is stator resistance online identification speed estimation. The stator resistance online identification model is set to operate after 10 minutes and updates resistance value due to the effect of temperature rise and corrects voltage model error to ensure the accuracy of the speed estimation.

\section{Simulation Analysis}

In order to verify the accuracy of the speed estimation method, the system simulation model is built by MATLAB software. Fig. 3 is the system simulation diagram. The motor's parameters are as follows: Power is $1.1 \mathrm{~kW}$; Rated voltage is $380 \mathrm{~V}$; Rated current is $2.5 \mathrm{~A}$; Rated frequency is $50 \mathrm{~Hz}$; Rated speed is $2825 \mathrm{r} / \mathrm{min}$; Pole pair is 1; Stator resistance is $5.27 \Omega$; Stator leakage inductance is $0.029 \mathrm{mH}$; Rotor resistance is $5.07 \Omega$; Rotor leakage inductance is $0.029 \mathrm{mH}$; Mutual inductance is $0.394 \mathrm{mH}$; Moment of inertia is $0.02 \mathrm{~kg} \cdot \mathrm{m}^{2}$.

The setting of proportion factor and integral factor is difficult, it must be determined by many 
experiments. When motor parameters are constant, PI adjustment factors are set as three different values, the no-load speed estimation waveform is shown in Fig.4.

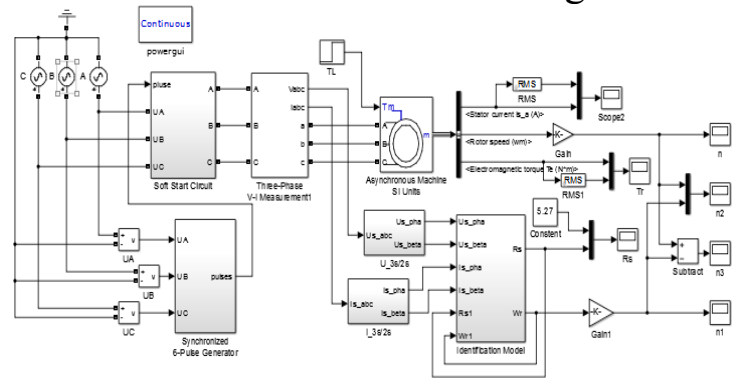

Fig. 3 Simulation system diagram

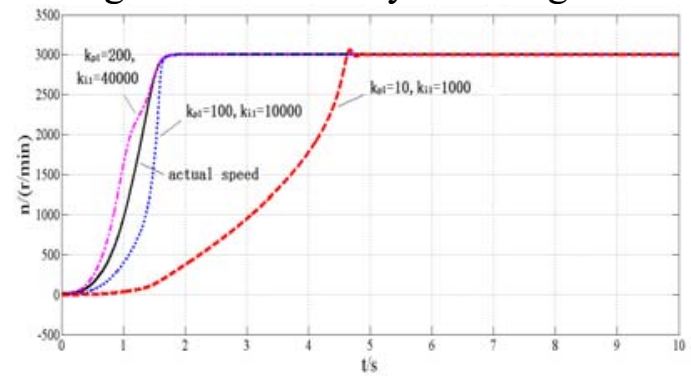

Fig. 4 Speed estimation waveform under different PI values

Following the trend of speed waveform in Fig.4, the simulation experiments finally determine $k_{p 1}=100, k_{i 1}=23800$. Fig.5 (a) is no-load speed waveform, Fig.5 (b) is load speed waveform after starting $2 \mathrm{~s}$ and adding $5 \mathrm{~N} \cdot \mathrm{m}$ load. The improved model is accurate, which achieves almost no static error in the steady state, and it can also converge to the actual value reliably with the load. All of the above verifies the applicability of the improved model in the speed estimation.

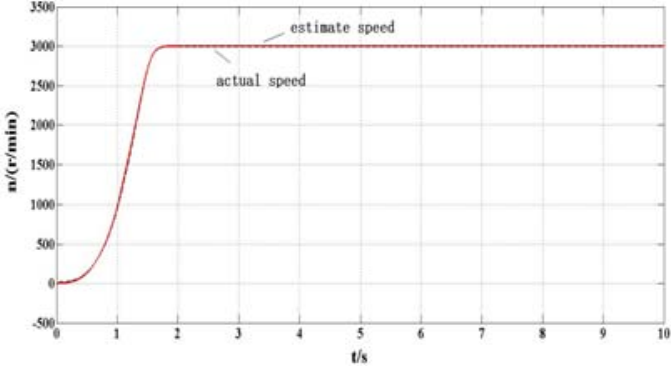

(a) No-load speed estimation wavefor

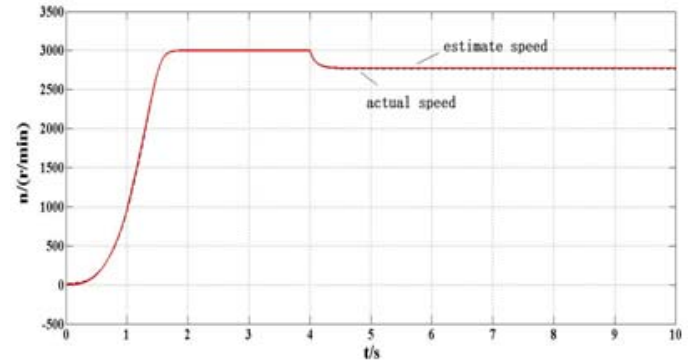

(b) Load speed estimation waveform

Fig. 5 Speed estimation waveform

It is assumed that the actual stator resistance is 2 times as much as the original, $\mathrm{Rs}=10.54 \Omega$. Fig 6 shows speed estimation results unrecognized resistance when the motor is stable. Fig.6(a) is the traditional MRAS speed estimation waveform. Fig.6(b) is the improved MRAS speed estimation waveform. The traditional MRAS not only has a great error, but also has a lot of low frequency oscillations, which seriously affect the stability of the system. The reason is that the pure integration process in traditional voltage model makes stator resistance continuously accumulate the error, and finally leads to divergence. Compared with the traditional model, the improved model has been greatly improved in the performance of speed regulation and has certain disturbance rejection ability, which can effectively eliminate or weaken the low frequency signal. But in Fig.6(b), the estimated speed is lower than the actual value, and there is a constant error which is about $5 \mathrm{r} / \mathrm{min}$. 

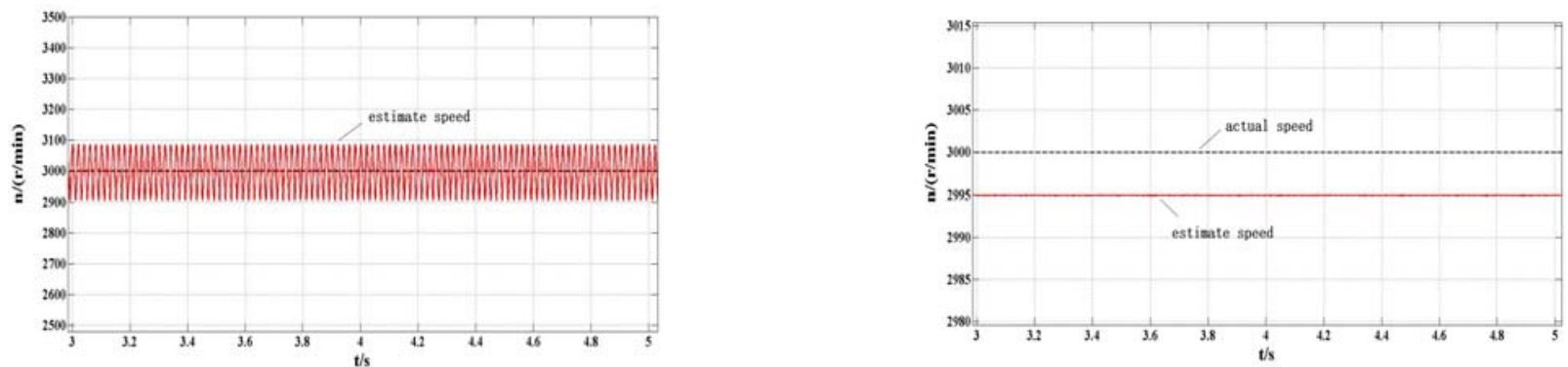

(a)Traditional MRAS speed estimation waveform (b) Improved MRAS speed estimation waveform

Fig. 6 Speed estimation waveform without resistance identification

In order to improve the accuracy of speed estimation, the stator resistance is identified online. Because there is no way to set the motor parameters in the process of simulation, it can be only simulated by changing the stator resistance value in the motor model. Fig.7 shows the identification results based on several stator resistance values. After numerous tests, it ensures $k_{p 2}=1, k_{i 2}=300$. It can be seen that the stator resistance basically converge to the true value in the $2 \mathrm{~s} \sim 3 \mathrm{~s}$. It shows that the method has a much better identification effect.

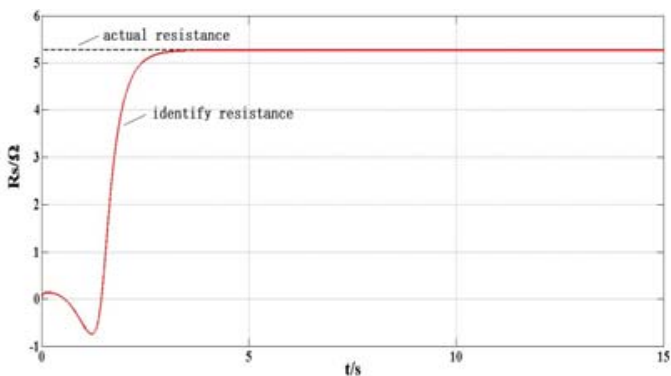

(a) $\mathrm{Rs}=5.27 \Omega$

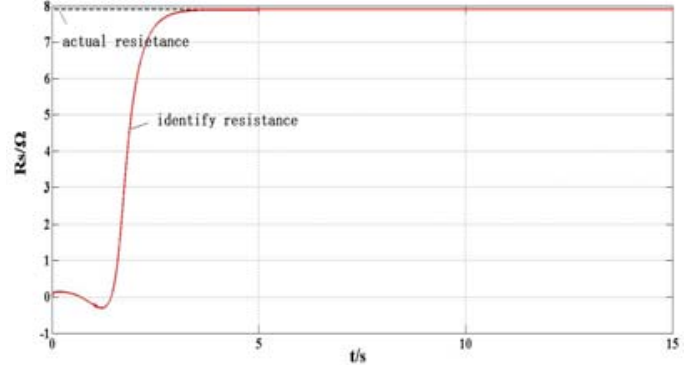

(b) $1.5 \mathrm{Rs}=7.905 \Omega$

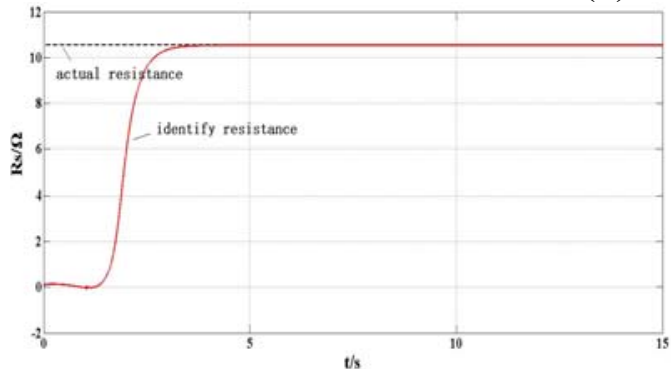

(c) $2 \mathrm{Rs}=10.54 \Omega$

Fig. 7 Stator resistance identification results

Stator resistance is 2 times as much as the original in Fig.8. Compared with Fig 6(b), the speed estimation accuracy is improved and the steady-state error is about $0.2 \mathrm{r} / \mathrm{min}$.

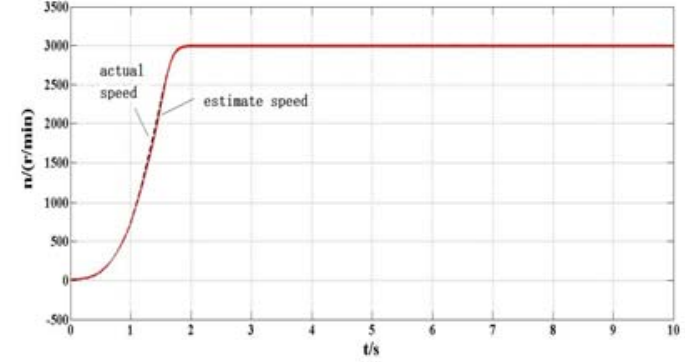

(a) Complete speed estimation waveform

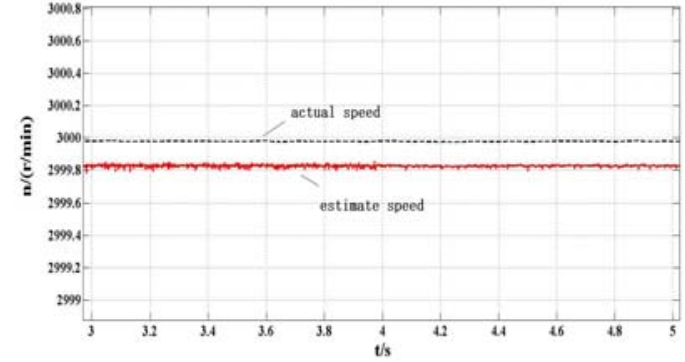

(b) Local amplification speed estimation waveform Fig. 8 Results of speed estimation with stator resistance online identification

\section{Experiment Verification}

DSPIC6014A is used as the control core of the experimental platform to verify the validity of the speed estimation system. Main circuit adopts soft starting circuit, which is composed of a three 
phase anti-parallel thyristor. Control circuit mainly includes: synchronous signal detection circuit, current sampling circuit, voltage sampling circuit, thyristor drive circuit, contactor control circuit, buttons and display. Control system block diagram is shown in Fig. 9.

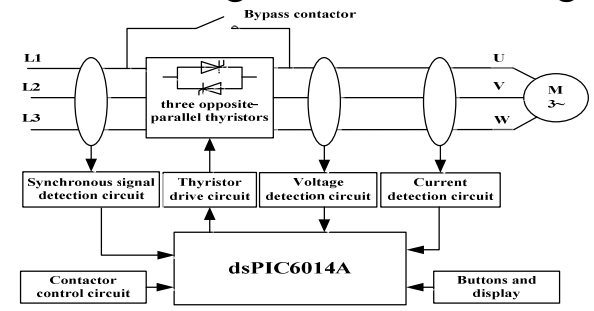

Fig. 9 Control system block diagram

It collects three-phase stator voltage and current signals and obtains digital quantity by $\mathrm{A} / \mathrm{D}$ converter, which are converted to two-phase stationary coordinate system. Then the voltage and current are recorded as the input of speed estimation system, the speed estimation value is received.

In the start process of soft starter, current and voltage waveform is shown in Fig10(a), in which three curves respectively represents the stator phase voltage RMS, stator current RMS and instantaneous value. Soft start controller running is stable, the starting current is small according to the above. No-load start-up speed compared with estimated speed curve as shown in Fig10(b). According to the experimental results, estimated speed fluctuates near the actual speed within a narrow range, so the speed estimation method in this paper is effectiveness and good performance.

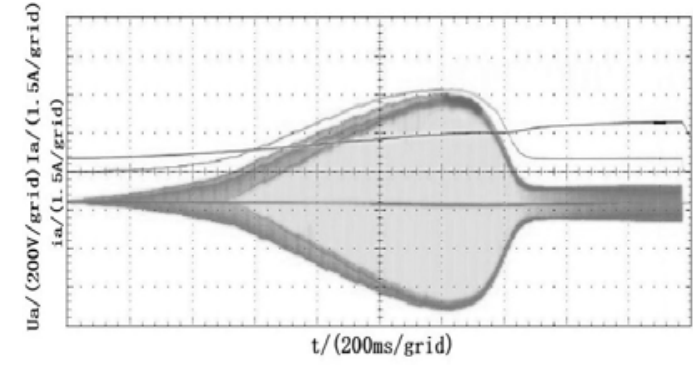

(a) Current and voltage waveform

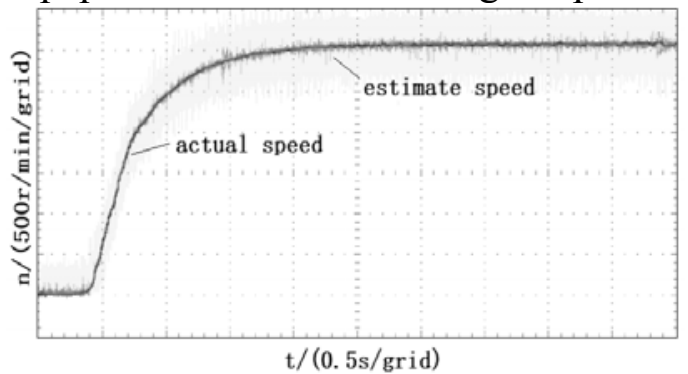

(b) Speed estimation waveform

Fig. 10 Experiment waveform

\section{Summary}

The traditional MRAS speed estimation method has the problems of integral initial value setting and DC drift and stator resistance variation at the low speed, which leads to speed estimation error. In regard to the shortcomings of traditional models, this paper makes improvements and designs a MRAS speed estimation method of stator resistance online identification. By MATLAB simulation and experiments, it is proved that this method can identify the stator resistance online and effectively eliminate the adverse effect of the pure integral and have strong robustness. The method has a certain reference value for induction motor speed estimation research and speed sensor-less control system application.

\section{References}

[1] Wei Zhu, Yong-hong Wang, Bo-shi Chen. Induction motor with stator resistance self-tuning MRAS speed estimation research[J]. Electric drive, 2014, 44 (12) : 8-11.

[2] Kai Xu. An interactive model parameter adaptive induction motor parameter online identification method research [J]. Journal of micromotor, 2008, 9 (2) : 15-18.

[3] Li-jun Zhang, Yi Ruan Xiao-xin Wu etc. MRAS speed estimation scheme based on stator current [J]. Journal of electrical and control applications, 2014, 9 (1) : 6-9.

[4] Ming-hui Chen,Yi Ruan, Jian Zong. Without speed sensor in the application of vector control system for mine electric locomotive[J]. Journal of motor and control applications, 2012,39 (2) : $17-20$. 
[5] Zhong-gang Yin, Jing Liu, Yan-ru Zhong, etc. Based on the double parameters of model reference adaptive speed sensor-less vector control of induction motor at low speed performance [J]. Electric Journal of engineering technology, 2012, 27 (7) : 124-130.

[6] Ming-yu Wang, Yang-yu Chen,Wei Deng, etc. The rotor resistance online identification of induction motor speed estimation method [J]. Journal of motor and control, 2010, 14 (4) : 66-71. 